Review began 10/10/2021 Review ended 10/14/2021 Published 10/16/2021

๑) Copyright 2021

Anwarali Khan et al. This is an open access article distributed under the terms of the Creative Commons Attribution License CCBY 4.0., which permits unrestricted use, distribution, and reproduction in any medium, provided the original author and source are credited.

\section{COVID-19 Collateral Damage: Management of Periprosthetic Joint Infection in Malaysia}

Mohammed Harris Anwarali Khan ${ }^{1}$, Ren Yi Kow ${ }^{2}$, Sasidaran Ramalingam ${ }^{1}$, Jade Pei Yuik Ho ${ }^{1}$, Jeffrey Jaya Raj $^{3}$, Kunalan Ganthel@Annamalai ${ }^{1}$, Chooi Leng Low ${ }^{4}$

1. Department of Orthopaedics, Hospital Kuala Lumpur, Kuala Lumpur, MYS 2. Department of Orthopaedics, Traumatology \& Rehabilitation, International Islamic University Malaysia, Kuantan, MYS 3. Department of Orthopaedics, Hospital Tengku Ampuan Afzan, Kuantan, MYS 4. Department of Radiology, International Islamic University Malaysia, Kuantan, MYS

Corresponding author: Ren Yi Kow, renyi_kow@hotmail.com

\section{Abstract}

\section{Background and objective}

Periprosthetic joint infection (PJI) is one of the dreaded complications in patients after arthroplasty surgeries, owing to the risk of morbidity and arduous investigations and management associated with it. Nevertheless, as Malaysia is currently battling against the severe acute respiratory syndrome coronavirus 2 (SARS-CoV-2)-induced coronavirus disease 2019 (COVID-19) pandemic head-on, the treatment for other non-life-threatening diseases including PJI has taken a backseat. In this study, we present a case series of 11 patients with PJI who were managed surgically at the largest tertiary hospital in Malaysia and we hope to shed some light on the difficulties we have encountered during this trying period.

\section{Patients and methods}

Patients with PJIs who underwent surgical intervention during the ongoing COVID-19 pandemic (March 1, 2020, to June 30, 2021) were reviewed and included in this study. The demographic profile of the patients, presenting complaints, prosthesis topography, biochemical investigative findings, surgical interventions, and short-term outcomes were summarized.

\section{Results}

A total of 11 patients were treated surgically at Hospital Kuala Lumpur for PJI. Among them, five patients are still awaiting their second-stage surgeries despite the completion of their antibiotic regimes, and they are fit for the procedure.

\section{Conclusion}

The COVID-19 pandemic has wreaked havoc on the treatment of patients with PJI. In a setting with scarce resources, surgeons should strongly consider single-stage revision surgeries for the treatment of patients with PJI.

Categories: Pathology, Infectious Disease, Orthopedics

Keywords: periprosthetic joint infection, covid 19, implant failure, infection, revision surgery

\section{Introduction}

As the severe acute respiratory syndrome coronavirus 2 (SARS-CoV-2)-induced coronavirus disease 2019 (COVID-19) pandemic sweeps through the whole world like a wildfire, crumbling most of the healthcare and other crucial sectors along the way, many countries are scrambling to manage and contain this debilitating disease [1-3]. As the healthcare system is stretched to the brink of collapse in many countries, the management of COVID-19 patients has taken precedence over other patients [1-3]. Elective surgical interventions have been postponed indefinitely, ranging from those for patients with cardiac diseases to patients with surgical illnesses [1-3]. While the administrators are justified in focusing on COVID-19 infections and associated mortality rates, the collateral damage of COVID-19 infection in the healthcare sector remains obscured to the public.

In Malaysia, elective surgeries such as joint replacement surgeries have been postponed throughout this pandemic period in many tertiary centers. Nevertheless, complications of joint replacement surgeries, such as periprosthetic joint infection (PJI), are considered to be orthopaedic emergencies, which require urgent surgical intervention. In patients who undergo primary joint replacement, the infection rate in the first two years usually ranges from 0.5 to $2 \%$ and revision surgeries normally have higher rates of infection, ranging from 2.5 to $4 \%$ [4,5]. PJI is a devastating condition associated with a high rate of mortality, increased risk of morbidity, decreased quality of life, and potentially decreased level of mobility and ambulation 


\section{Cureus}

$[4,5]$. Common risk factors for joint replacement surgery are postoperative surgical site infection, previous arthroplasty, advanced age, malnutrition, joint disease, obesity, diabetes mellitus, and remote infection [46]. Management of PJI can be done via single- or two-stage revision surgery, with each having its pros and cons $[7,8]$. During the COVID-19 pandemic, surgeons have to improvise and adjust accordingly, depending on the surge of patients infected with the SARS-CoV- 2 virus. We present a case series of 11 patients with PJI who were managed surgically during the ongoing pandemic at the largest tertiary hospital in Malaysia and we hope to highlight the difficulties we have encountered during this trying period.

\section{Materials And Methods}

All patients who were treated surgically at Hospital Kuala Lumpur from March 1, 2020, to June 30, 2021, for PJI were included in this study. Written consents were obtained from all patients for the publication of this article. They were diagnosed to have PJI based on the Musculoskeletal Infection Society (MSIS) criteria, whereby at least one of the following criteria was present (Table 1) [8].

Periprosthetic joint infection diagnosis based on the Musculoskeletal Infection Society (MSIS) criteria, whereby at least one of the following criteria was present

1 Presence of sinus tract communicating with the prosthesis

2 A microorganism was isolated from the affected joint from at least two separate tissues or synovial fluid samples

Four out of six following criteria were fulfilled

A Raised serum erythrocyte sedimentation rate (ESR) and serum C-reactive protein (CRP)

B Elevated synovial white blood cell count

3 C Raised synovial neutrophil percentage

D Presence of pus in the affected joint

E Isolation of a pathogen in one culture of periprosthetic tissue or synovial fluid

F More than five neutrophils per high-power field from histologic analysis of affected joint tissue

TABLE 1: Periprosthetic joint infection diagnosis based on the Musculoskeletal Infection Society (MSIS) criteria

All patients were followed up for a minimum of four months after the surgical intervention. The demographic data, investigative results, and types of surgical interventions were obtained from the medical records. Patients who did not fulfill the MSIS criteria or those who were lost to follow-up were excluded from this case series.

\section{Results}

A total of 11 patients were enrolled in this study, with ages ranging from 39 to 79 years. Most patients were males $(n=7,63.6 \%)$ with Chinese being the most predominant ethnicity $(n=6,54.5 \%)$. All patients had preexisting comorbidities, with a majority of them having identifiable risk factors $(\mathrm{n}=9,81.8 \%)$. Most of the PJIs involved the knee joint ( $\mathrm{n}=7,63.6 \%)$, followed by the hip joint (three total hip replacements and one bipolar hemiarthroplasty). Their presenting complaint was mostly pain or pain with joint swelling with a variable duration ranging from two days to six months.

Table 2 summarizes the patients' demographics (age, gender, body mass index, smoking status, comorbidities, and other risk factors), their presenting complaints and duration, and the affected joint topography. 


\begin{tabular}{|c|c|c|c|c|c|c|c|c|c|c|c|c|}
\hline Patient & $\begin{array}{l}\text { Age } \\
\text { (years) }\end{array}$ & Gender & Ethnicity & $\left(\mathrm{kg} / \mathrm{m}^{2}\right)$ & $\begin{array}{l}\text { Smoking } \\
\text { status }\end{array}$ & Comorbidities & $\begin{array}{l}\text { Risk } \\
\text { factors }\end{array}$ & $\begin{array}{l}\text { Signs and } \\
\text { symptoms }\end{array}$ & $\begin{array}{l}\text { Duration } \\
\text { of } \\
\text { symptoms }\end{array}$ & $\begin{array}{l}\text { Duration } \\
\text { from } \\
\text { index } \\
\text { surgery }\end{array}$ & $\begin{array}{l}\text { Prosthesis } \\
\text { topography }\end{array}$ & $\begin{array}{l}\text { Initial } \\
\text { indication } \\
\text { for } \\
\text { replacement } \\
\text { surgery }\end{array}$ \\
\hline 1 & 74 & $\mathrm{~F}$ & Chinese & 30 & No & $\begin{array}{l}\text { DM, } \\
\text { hyperlipidemia, } \\
\text { rheumatoid } \\
\text { arthritis }\end{array}$ & $\begin{array}{l}\text { Steroid } \\
\text { treatment }\end{array}$ & $\begin{array}{l}\text { Right hip } \\
\text { pain }\end{array}$ & 6 months & 14 years & Right THR & Osteoarthritis \\
\hline 2 & 39 & $\mathrm{M}$ & Indian & 26.6 & No & $\begin{array}{l}\text { Rheumatoid } \\
\text { arthritis, } \\
\text { Cushing's } \\
\text { syndrome, } \\
\text { Hepatitis B }\end{array}$ & $\begin{array}{l}\text { Steroid } \\
\text { treatment, } \\
\text { hepatitis } \\
\text { B }\end{array}$ & $\begin{array}{l}\text { Right knee } \\
\text { pain with } \\
\text { sinus } \\
\text { discharge }\end{array}$ & 1 week & 6 years & Right TKR & Osteoarthritis \\
\hline 3 & 70 & $\mathrm{~F}$ & Chinese & 25 & No & Hypertension & $\begin{array}{l}\text { History of } \\
\text { cellulitis }\end{array}$ & $\begin{array}{l}\text { Right knee } \\
\text { pain and } \\
\text { swelling }\end{array}$ & 1 month & 2 years & Right TKR & Osteoarthritis \\
\hline 4 & 75 & M & Chinese & 27.7 & No & Hypertension & Nil & $\begin{array}{l}\text { Left knee } \\
\text { pain }\end{array}$ & 1 month & 2 years & Left TKR & Osteoarthritis \\
\hline 5 & 68 & $\mathrm{~F}$ & Indian & 24.2 & No & $\begin{array}{l}\text { Hypertension, } \\
\text { chronic renal } \\
\text { disease }\end{array}$ & $\begin{array}{l}\text { Chronic } \\
\text { renal } \\
\text { disease }\end{array}$ & $\begin{array}{l}\text { Right knee } \\
\text { swelling }\end{array}$ & 1 month & 4 years & Right TKR & Osteoarthritis \\
\hline 6 & 79 & $\mathrm{~F}$ & Chinese & 30 & No & $\begin{array}{l}\text { DM, } \\
\text { hypertension, } \\
\text { hyperlipidemia }\end{array}$ & DM & $\begin{array}{l}\text { Right knee } \\
\text { pain }\end{array}$ & 2 days & 1 year & Right TKR & Osteoarthritis \\
\hline 7 & 63 & M & Malay & 30 & No & $\begin{array}{l}\text { Hypertension, } \\
\text { chronic renal } \\
\text { disease }\end{array}$ & $\begin{array}{l}\text { Chronic } \\
\text { renal } \\
\text { disease }\end{array}$ & $\begin{array}{l}\text { Left hip } \\
\text { pain }\end{array}$ & 6 months & 10 years & Left THR & Osteoarthritis \\
\hline 8 & 66 & $\mathrm{~F}$ & Malay & 32 & No & Hypertension & $\begin{array}{l}\text { History of } \\
\text { cellulitis }\end{array}$ & $\begin{array}{l}\text { Right knee } \\
\text { pain }\end{array}$ & 2 weeks & 10 years & Right TKR & Osteoarthritis \\
\hline 9 & 74 & $\mathrm{~F}$ & Chinese & 16.6 & No & $\begin{array}{l}\text { Hypertension, } \\
\text { hyperlipidemia }\end{array}$ & $\begin{array}{l}\text { Delayed } \\
\text { suture } \\
\text { removal }\end{array}$ & $\begin{array}{l}\text { Left hip } \\
\text { pain and } \\
\text { swelling }\end{array}$ & 10 days & 2 months & $\begin{array}{l}\text { Left bipolar } \\
\text { hemiarthroplasty }\end{array}$ & $\begin{array}{l}\text { Neck of } \\
\text { femur } \\
\text { fracture }\end{array}$ \\
\hline 10 & 56 & M & Chinese & 28.4 & No & Hypertension & Nil & $\begin{array}{l}\text { Right hip } \\
\text { pain and } \\
\text { swelling }\end{array}$ & 3 months & 1 year & Right THR & Osteoarthritis \\
\hline 11 & 53 & $\mathrm{~F}$ & Malay & 30 & No & $\begin{array}{l}\text { DM, } \\
\text { hypertension }\end{array}$ & $\begin{array}{l}\text { Skin } \\
\text { infection }\end{array}$ & $\begin{array}{l}\text { Right knee } \\
\text { pain and } \\
\text { swelling }\end{array}$ & 3 months & 1 year & Right TKR & Osteoarthritis \\
\hline
\end{tabular}

TABLE 2: Patients' demographic data, risk factors, presenting complaints and duration, and the affected joint topography

M: male; F: female; BMI: body mass index; DM: diabetes mellitus; THR: total hip replacement; TKR: total knee replacement

Table 3 illustrates the patients' investigation results such as serum parameters (white cell count, C-reactive protein level, and erythrocyte sedimentation rate), plain radiographic findings, samples obtained and pathogens isolated, and their subsequent surgical intervention and chemotherapy details.

\begin{tabular}{|c|c|c|c|c|c|c|c|c|c|c|c|}
\hline Patient & $\begin{array}{l}\text { WBC } \\
\left(10^{9} / \mathrm{L}\right)\end{array}$ & $\begin{array}{l}\text { CRP } \\
\text { (mg/L) }\end{array}$ & $\begin{array}{l}\text { ESR } \\
(\mathrm{mm} / \mathrm{hr})\end{array}$ & $\begin{array}{l}\text { X-ray } \\
\text { findings }\end{array}$ & Sample(s) & $\begin{array}{l}\text { Microorganism } \\
\text { isolated }\end{array}$ & $\begin{array}{l}\text { First-stage } \\
\text { surgery }\end{array}$ & $\begin{array}{l}\text { Second- } \\
\text { stage } \\
\text { surgery }\end{array}$ & $\begin{array}{l}\text { Antibiotic } \\
\text { usage }\end{array}$ & $\begin{array}{l}\text { Short- } \\
\text { term } \\
\text { outcome }\end{array}$ & Comment \\
\hline & & & & Implant & & & & & & & \\
\hline
\end{tabular}




\section{Cureus}

\begin{tabular}{|c|c|c|c|c|c|c|c|c|c|c|c|}
\hline 1 & 7.9 & 3.7 & 47 & $\begin{array}{l}\text { loosening at } \\
\text { the } \\
\text { acetabulum } \\
\text { and femoral } \\
\text { component } \\
\text { with } \\
\text { osteomyelitis } \\
\text { at the } \\
\text { proximal } \\
\text { femur }\end{array}$ & $\begin{array}{l}\text { Synovial } \\
\text { fluid, } \\
\text { tissue, and } \\
\text { bone }\end{array}$ & No growth & $\begin{array}{l}\text { WD, IR, } \\
\text { and } \\
\text { cement } \\
\text { spacer with } \\
\text { interlocking } \\
\text { nail }\end{array}$ & Pending & $\begin{array}{l}\text { IV cloxacillin } \\
\text { for } 6 \text { weeks } \\
\text { then oral } \\
\text { cloxacillin } \\
\text { for } 3 \text { months }\end{array}$ & $\begin{array}{l}\text { The wound } \\
\text { healed, } \\
\text { wheelchair } \\
\text { ambulating }\end{array}$ & \\
\hline 2 & 10.7 & 6.7 & 43 & $\begin{array}{l}\text { No implant } \\
\text { loosening }\end{array}$ & $\begin{array}{l}\text { Tissue and } \\
\text { pus }\end{array}$ & ESBL & $\begin{array}{l}\text { WD, JW } \\
\text { (two times) }\end{array}$ & NA & $\begin{array}{l}\text { IV } \\
\text { meropenem } \\
\text { for } 6 \text { weeks }\end{array}$ & $\begin{array}{l}\text { The wound } \\
\text { healed, } \\
\text { ambulating } \\
\text { with a } \\
\text { walking } \\
\text { aid }\end{array}$ & $\begin{array}{l}\text { The } \\
\text { patient } \\
\text { refuses } \\
\text { the } \\
\text { removal of } \\
\text { the } \\
\text { implant }\end{array}$ \\
\hline 3 & 4.1 & 5.5 & 39 & $\begin{array}{l}\text { No implant } \\
\text { loosening }\end{array}$ & $\begin{array}{l}\text { Synovial } \\
\text { fluid }\end{array}$ & No growth & $\begin{array}{l}\text { WD, JW, } \\
\text { and insert } \\
\text { exchange }\end{array}$ & NA & $\begin{array}{l}\text { IV } \\
\text { ciprofloxacin } \\
\text { and } \\
\text { clindamycin } \\
\text { for } 1 \text { week } \\
\text { then oral } \\
\text { Bactrim and } \\
\text { rifampicin } \\
\text { for } 3 \text { months }\end{array}$ & $\begin{array}{l}\text { The wound } \\
\text { healed, } \\
\text { ambulating } \\
\text { without } \\
\text { walking } \\
\text { aid }\end{array}$ & \\
\hline 4 & 9.3 & 56.6 & 115 & $\begin{array}{l}\text { No implant } \\
\text { loosening }\end{array}$ & $\begin{array}{l}\text { Synovial } \\
\text { fluid }\end{array}$ & CONS & $\begin{array}{l}\text { WD, JW, } \\
\text { and } \\
\text { cement } \\
\text { spacer }\end{array}$ & Pending & $\begin{array}{l}\text { IV } \\
\text { vancomycin } \\
\text { for } 2 \text { weeks } \\
\text { then oral } \\
\text { Bactrim and } \\
\text { clindamycin } \\
\text { for } 3 \text { months }\end{array}$ & $\begin{array}{l}\text { The wound } \\
\text { healed, } \\
\text { ambulating } \\
\text { without } \\
\text { walking } \\
\text { aid }\end{array}$ & \\
\hline 5 & 7.7 & 105 & 117 & $\begin{array}{l}\text { No implant } \\
\text { loosening }\end{array}$ & $\begin{array}{l}\text { Synovial } \\
\text { fluid, } \\
\text { tissue, and } \\
\text { bone }\end{array}$ & $\begin{array}{l}\text { Acinetobacter } \\
\text { baumanii }\end{array}$ & $\begin{array}{l}\text { WD, JW, } \\
\text { and } \\
\text { cement } \\
\text { spacer }\end{array}$ & Pending & $\begin{array}{l}\text { IV } \\
\text { Augmentin } \\
\text { for } 2 \text { weeks } \\
\text { then oral } \\
\text { Unasyn for } \\
3 \text { months }\end{array}$ & $\begin{array}{l}\text { The wound } \\
\text { healed, } \\
\text { partially } \\
\text { ambulating } \\
\text { with a } \\
\text { walking } \\
\text { aid }\end{array}$ & \\
\hline 6 & 8 & 3.1 & 63 & $\begin{array}{l}\text { No implant } \\
\text { loosening }\end{array}$ & $\begin{array}{l}\text { Synovial } \\
\text { fluid }\end{array}$ & $\begin{array}{l}\text { Staphylococcus } \\
\text { aureus }\end{array}$ & $\begin{array}{l}\text { WD, JW, } \\
\text { and } \\
\text { cement } \\
\text { spacer }\end{array}$ & NA & $\begin{array}{l}\text { IV cloxacillin } \\
\text { for } 2 \text { weeks } \\
\text { then oral } \\
\text { cloxacillin } \\
\text { and fusidic } \\
\text { acid for } 6 \\
\text { weeks }\end{array}$ & $\begin{array}{l}\text { The wound } \\
\text { healed, } \\
\text { ambulating } \\
\text { without } \\
\text { walking } \\
\text { aid }\end{array}$ & \\
\hline 7 & 9 & 3.4 & 26 & $\begin{array}{l}\text { No implant } \\
\text { loosening }\end{array}$ & $\begin{array}{l}\text { Synovial } \\
\text { fluid }\end{array}$ & CONS & $\begin{array}{l}\text { WD, JW, } \\
\text { and insert } \\
\text { exchange }\end{array}$ & Pending & $\begin{array}{l}\text { IV } \\
\text { clindamycin } \\
\text { for } 2 \text { weeks } \\
\text { then oral } \\
\text { Unasyn for } \\
3 \text { months }\end{array}$ & $\begin{array}{l}\text { The wound } \\
\text { healed, } \\
\text { ambulating } \\
\text { with non- } \\
\text { weight- } \\
\text { bearing } \\
\text { clutches }\end{array}$ & \\
\hline 8 & 10 & 161 & 120 & $\begin{array}{l}\text { Implant } \\
\text { loosening at } \\
\text { the femoral } \\
\text { and tibial } \\
\text { component }\end{array}$ & $\begin{array}{l}\text { Synovial } \\
\text { fluid }\end{array}$ & $\begin{array}{l}\text { Streptococcus } \\
\text { dysgalactiae }\end{array}$ & $\begin{array}{l}\text { WD, JW, } \\
\text { and insert } \\
\text { exchange }\end{array}$ & NA & $\begin{array}{l}\text { IV penicillin } \\
\text { for } 2 \text { weeks } \\
\text { then oral } \\
\text { Unasyn for } \\
10 \text { weeks }\end{array}$ & $\begin{array}{l}\text { The wound } \\
\text { healed, } \\
\text { ambulating } \\
\text { with a } \\
\text { walking } \\
\text { aid }\end{array}$ & \\
\hline & & & & & & & & & & The wound & \\
\hline
\end{tabular}




\section{Cureus}

\begin{tabular}{|c|c|c|c|c|c|c|c|c|c|c|}
\hline 9 & 8.86 & 20 & 66 & $\begin{array}{l}\text { No implant } \\
\text { loosening }\end{array}$ & $\begin{array}{l}\text { Synovial } \\
\text { fluid }\end{array}$ & $\begin{array}{l}\text { Gram-positive } \\
\text { bacilli }\end{array}$ & $\begin{array}{l}\text { WD, JW, } \\
\text { and } \\
\text { Collatamp } \\
\text { insertion }\end{array}$ & NA & $\begin{array}{l}\text { Oral Bactrim } \\
\text { and oral } \\
\text { doxycycline } \\
\text { for } 6 \text { months }\end{array}$ & $\begin{array}{l}\text { healed, } \\
\text { ambulating } \\
\text { with a } \\
\text { walking } \\
\text { aid }\end{array}$ \\
\hline 10 & 7.7 & 84 & 106 & $\begin{array}{l}\text { No implant } \\
\text { loosening }\end{array}$ & Tissue & Serratia sp. & $\begin{array}{l}\text { WD, JW, } \\
\text { and } \\
\text { exchange } \\
\text { of femoral } \\
\text { head } \\
\text { component }\end{array}$ & NA & $\begin{array}{l}\text { IV cefepime } \\
\text { for } 6 \text { weeks } \\
\text { then oral } \\
\text { ciprofloxacin } \\
\text { for } 6 \text { weeks }\end{array}$ & $\begin{array}{l}\text { The wound } \\
\text { healed, } \\
\text { ambulating } \\
\text { with a } \\
\text { walking } \\
\text { aid }\end{array}$ \\
\hline 11 & 10 & 35 & 117 & $\begin{array}{l}\text { No implant } \\
\text { loosening }\end{array}$ & $\begin{array}{l}\text { Synovial } \\
\text { fluid }\end{array}$ & $\begin{array}{l}\text { Staphylococcus } \\
\text { aureus }\end{array}$ & $\begin{array}{l}\text { WD, IR, } \\
\text { JW, and } \\
\text { cement } \\
\text { spacer }\end{array}$ & Pending & $\begin{array}{l}\text { IV cloxacillin } \\
\text { for } 2 \text { weeks } \\
\text { then oral } \\
\text { cloxacillin } \\
\text { for } 3 \text { months }\end{array}$ & $\begin{array}{l}\text { The wound } \\
\text { healed, } \\
\text { partially } \\
\text { ambulating } \\
\text { with a } \\
\text { walking } \\
\text { aid }\end{array}$ \\
\hline
\end{tabular}

TABLE 3: Patients' investigation results, surgical intervention and antibiotic therapy, and their short-term outcomes

ESBL: extended-spectrum beta-lactamase; CONS: coagulase-negative staphylococci; WD: wound debridment; IR: implant removal; JW: joint washout; IV: intravenous; Augmentin: amoxicillin/clavulanate; Bactrim: trimethoprim/sulfamethoxazole; Unasyn: ampicillin/sulbactam

Most of the patients' serum white blood cell counts were not elevated, ranging from 4.1 to $10.7 \times 10^{9} / \mathrm{L}$. Similarly, only five patients (45.4\%) had raised serum C-reactive protein. Nevertheless, all patients except one had elevated erythrocyte sedimentation rates. Upon reviewing the plain radiographs, only two patients had signs to suggest implant loosening affecting the hip and knee joints respectively. In terms of sample collection, no antibiotic was given two weeks prior to the collection of intraoperative cultures as suggested by the American Academy of Orthopaedic Surgeons (AAOS) [9]. Tissue and bone samples were directly sent to the laboratory while synovial fluid samples were sent in blood culture bottles $\left(\right.$ BACTEC $^{\mathrm{TM}}$, Becton Dickinson Diagnostic Instrument Systems, Sparks, MD). All except two samples yielded positive cultures with various pathogens isolated.

Five patients (45.4\%) underwent wound debridement, implant removal, and antibiotic cement spacer insertion, while another five patients (45.4\%) underwent single-stage surgeries. One patient refused implant removal and underwent wound debridement and joint washout twice. Despite the completion of antibiotics with subsequent improvement in septic parameters, their second-stage surgeries were postponed indefinitely due to the COVID-19 pandemic affecting the availability of operation theatres. Among the patients who underwent single-stage surgeries, two were able to ambulate without a walking aid while another three patients were ambulating with a walking aid.

\section{Discussion}

PJI remains a significant challenge for arthroplasty surgeons in terms of diagnosis as well as management [9]. A combination of detailed clinical findings, blood and radiological investigations, coupled with synovial or tissue culture are required to properly diagnose a PJI. In the midst of the ongoing COVID-19 pandemic, patients with SARS-CoV-2 infection can present with atypical symptoms masquerading as PJI to further complicate the process of PJI diagnosis [10]. The dilemma we encounter was represented by case 8 in this series, wherein the patient who had undergone a bilateral total knee replacement 11 years ago presented with fever for one day associated with right knee pain and swelling for two weeks. She had a history of right lower limb cellulitis requiring admission and intravenous antibiotics two years ago. Clinically, her right knee was warm and tender with signs of effusion and limited range of movement. Her septic parameters were all elevated, further suggesting a PJI. Nevertheless, she had been in close contact with a patient with the COVID-19 infection, making her a person-under-investigation (PUI). While waiting for her polymerase chain reaction (PCR) result, arthrocentesis from the affected joint yielded Gram-positive pathogens. As she was relatively stable, she was scheduled for surgery after the period of quarantine and a repeat PCR test. She subsequently underwent wound debridement, synovectomy, joint washout, and polyethylene insert exchange.

Besides the admitted patients being at risk of COVID-19 infection, as the COVID-19 pandemic stretches the healthcare resources to the brink of collapse, most of the hospitals place their focus solely on treating patients with life-threatening conditions. All elective surgeries have been postponed to channel the 


\section{Cureus}

resources, in terms of manpower, bed availability, and medications, toward the management of patients with SARS-CoV-2 infection. This has created an imbroglio, wherein patients who have undergone first-stage surgery and have completed antibiotic chemotherapy have to wait indefinitely for the second-stage surgery as COVID-19 shows no sign of abating. Throughout this pandemic period, there have been five patients in our center who are still awaiting their second-stage surgeries despite the completion of their antibiotic regimens and although their septic parameters are appropriate for second-stage surgery. Patients who have had first-stage revision are initially planned for revision surgeries six weeks after their first-stage surgeries. In view of the delay in the second-stage surgery, we have decided to stop antibiotics three months after the first-stage surgery despite the fact that the routine practice in our center is to continue antibiotics until the second-stage surgery. A comparative analysis of different issues encountered during the COVID-19 pandemic versus the pre-pandemic period regarding the management of patients with PJI is presented in Table 4.

\begin{tabular}{|c|c|c|}
\hline $\begin{array}{l}\text { Issues } \\
\text { encountered }\end{array}$ & Pre-pandemic period & During the pandemic \\
\hline $\begin{array}{l}\text { Patients } \\
\text { presentation }\end{array}$ & Early (usually within days to weeks upon symptoms occurrence) & Delayed (mean of 60 days in this series) \\
\hline Investigations & $\begin{array}{l}\text { Routine septic investigations (biochemical, culture, and radiographic } \\
\text { investigations) }\end{array}$ & $\begin{array}{l}\text { Routine septic investigations PLUS COVID-19 } \\
\text { polymerase chain reaction (PCR) }\end{array}$ \\
\hline $\begin{array}{l}\text { Bed } \\
\text { management }\end{array}$ & Generally separated from patients who have undergone clean surgeries & $\begin{array}{l}\text { Generally separated from patients with PUI status. } \\
\text { Separation from patients with clean surgeries cannot be } \\
\text { guaranteed }\end{array}$ \\
\hline $\begin{array}{l}\text { Management } \\
\text { (first-stage } \\
\text { surgery) }\end{array}$ & $\begin{array}{l}\text { Options of wound debridement, joint washout, and insert exchange or } \\
\text { implant removal and cement spacer insertion, depending on } \\
\text { intraoperative findings }\end{array}$ & $\begin{array}{l}\text { Single-stage surgery such as wound debridement, joint } \\
\text { washout, and insert exchange is preferred if clinically } \\
\text { indicated }\end{array}$ \\
\hline $\begin{array}{l}\text { Management } \\
\text { (antibiotic } \\
\text { regimen) }\end{array}$ & $\begin{array}{l}\text { Generally given for } 6 \text { to } 8 \text { weeks until second-stage surgery (based on } \\
\text { culture and sensitivity) }\end{array}$ & $\begin{array}{l}\text { Given for a maximum of } 3 \text { months (while waiting for } \\
\text { second-stage surgery) }\end{array}$ \\
\hline $\begin{array}{l}\text { Management } \\
\text { (second-stage } \\
\text { surgery) }\end{array}$ & $\begin{array}{l}\text { Performed when clinically indicated (favorable soft tissue conditions and } \\
\text { improved septic parameters) }\end{array}$ & Postponed indefinitely due to the COVID-19 pandemic \\
\hline
\end{tabular}

TABLE 4: Comparison of different issues encountered during the COVID-19 pandemic and prepandemic period for the management of patients with PJI

PJI: periprosthetic joint infection; COVID-19: coronavirus disease 2019; PUI: person under investigation

In this difficult time, the question remains as to how to optimize the recovery of patients with PJI without increasing the burden on the healthcare system. This prompts us to favor single-stage revision surgery instead of the cumbersome two-stage revision surgery. There is now evidence from several comparative case series that complications and reinfection rates after single-stage revisions are similar to those in two-stage revisions [11-14]. Revision surgery protocols of PJI, designed to address the nature of these infections, were first introduced over three decades ago. The fundamental concepts for the treatment of PJI entail the necessity for implants, foreign bodies, and cement removal in combination with radical debridement. This is coupled with the use of antibiotic-loaded cement. It is based on these principles that today's limb salvage protocols have evolved. Surgeons are now able to take advantage of growing technological innovations and employ aggressive surgical techniques for debridement followed by the restoration of function in patients with PJI. The surge in COVID-19 cases has shortened the operation list and surgery time is significantly reduced. Wise decisions should be made and resources available should be utilized smartly and judiciously to provide the best care to patients. In our center, those who underwent single-stage surgeries have achieved relatively good short-term outcomes.

\section{Limitations of the study}

This study was limited by the small number of patients included from a single institution. Nevertheless, this was the first-of-its-kind study to shed some light on an important aspect of the COVID-19 collateral damage.

\section{Conclusions}


The COVID-19 pandemic has caused unmeasurable collateral damage to the healthcare system globally. Many patients suffering from non-COVID-19-related diseases have received sub-standard treatment or have had their treatment delayed. It is paramount for healthcare workers to be equipped with the relevant knowledge and clinical wisdom in treating patients during this COVID-19 pandemic. Surgeons should always maintain the highest clinical awareness and level of suspicion in terms of recognizing signs and symptoms of PII. In the midst of the COVID-19 pandemic, surgeons should strongly consider the option of single-stage surgery for patients with PJI whenever feasible.

\section{Additional Information \\ Disclosures}

Human subjects: Consent was obtained or waived by all participants in this study. Animal subjects: All authors have confirmed that this study did not involve animal subjects or tissue. Conflicts of interest: In compliance with the ICMJE uniform disclosure form, all authors declare the following: Payment/services info: All authors have declared that no financial support was received from any organization for the submitted work. Financial relationships: All authors have declared that they have no financial relationships at present or within the previous three years with any organizations that might have an interest in the submitted work. Other relationships: All authors have declared that there are no other relationships or activities that could appear to have influenced the submitted work.

\section{References}

1. Ball J, Nehme Z, Bernard S, Stub D, Stephenson M, Smith K: Collateral damage: hidden impact of the COVID-19 pandemic on the out-of-hospital cardiac arrest system-of-care. Resuscitation. 2020, 156:157-63. 10.1016/j.resuscitation.2020.09.017

2. Emanuel EJ, Persad G, Upshur R, et al.: Fair allocation of scarce medical resources in the time of Covid-19 . N Engl J Med. 2020, 382:2049-55. 10.1056/NEJMsb2005114

3. Kibbe MR: Surgery and COVID-19. JAMA. 2020, 324:1151-2. 10.1001/jama.2020.15191

4. Aslam S, Darouiche RO: Prosthetic joint infections. Curr Infect Dis Rep. 2012, 14:551-7. 10.1007/s11908012-0284-2

5. Abad CL, Haleem A: Prosthetic joint infections: an update. Curr Infect Dis Rep. 2018, 20:15. 10.1007/s11908-018-0622-0

6. Bongartz T, Halligan CS, Osmon DR, et al.: Incidence and risk factors of prosthetic joint infection after total hip or knee replacement in patients with rheumatoid arthritis. Arthritis Rheum. 2008, 59:1713-20. 10.1002/art.24060

7. Kunutsor SK, Whitehouse MR, Blom AW, et al.: One- and two-stage surgical revision of peri-prosthetic joint infection of the hip: a pooled individual participant data analysis of 44 cohort studies. Eur J Epidemiol. 2018, 33:933-46. 10.1007/s10654-018-0377-9

8. Aggarwal VK, Rasouli MR, Parvizi J: Periprosthetic joint infection: current concept. Indian J Orthop. 2013, 47:10-7. 10.4103/0019-5413.106884

9. Tubb CC, Polkowksi GG, Krause B: Diagnosis and prevention of periprosthetic joint infections . J Am Acad Orthop Surg. 2020, 28:e340-8. 10.5435/JAAOS-D-19-00405

10. Kenanidis E, Kakoulidis P, Anagnostis P, Beletsiotis A, Tsiridis E: Coronavirus disease 2019 (COVID-19) can masquerade as acute postoperative periprosthetic joint infection. Cureus. 2020, 12:e7857. 10.7759 /cureus. 7857

11. Klemt C, Tirumala V, Oganesyan R, Xiong L, van den Kieboom J, Kwon YM: Single-stage revision of the infected total knee arthroplasty is associated with improved functional outcomes: a propensity scorematched cohort study. J Arthroplasty. 2021, 36:298-304. 10.1016/j.arth.2020.07.012

12. Pangaud C, Ollivier M, Argenson JN: Outcome of single-stage versus two-stage exchange for revision knee arthroplasty for chronic periprosthetic infection. EFORT Open Rev. 2019, 4:495-502. 10.1302/20585241.4.190003

13. van den Kieboom J, Tirumala V, Box H, Oganesyan R, Klemt C, Kwon YM: One-stage revision is as effective as two-stage revision for chronic culture-negative periprosthetic joint infection after total hip and knee arthroplasty. Bone Joint J. 2021, 103-B:515-21. 10.1302/0301-620X.103B.BJJ-2020-1480.R2

14. Yeung CM, Suhardi VJ, Varady NH, et al.: Trends of prosthetic joint infection organisms and recurrence for a single high-volume arthroplasty surgeon over 20 years. J Arthroplasty. 2021, 36:1101-8. 10.1016/j.arth.2020.10.002 